\title{
The Role of veA in Aspergillus flavus Infection of Peanut, Corn and Cotton
}

\author{
R.M. Duran ${ }^{1}$, J.W. Cary ${ }^{2}$, A.M. Calvo ${ }^{*}, 1$ \\ ${ }^{I}$ Department of Biological Sciences, Northern Illinois University, Dekalb, IL, USA \\ ${ }^{2}$ Southern Regional Research Center, Agricultural Research Service, U.S. Department of Agriculture, New Orleans, LA, \\ USA
}

\begin{abstract}
The aflatoxin-producing fungus Aspergillus flavus is a causal agent of preharvest contamination of food commodities such as oil seed crops worldwide. Peanut, corn and cottonseed are among the oil seeds that are susceptible to aflatoxin contamination during invasion of these crops by A. flavus. Contamination of agricultural commodities with aflatoxins can result in serious economic hardships to producers and adverse health impacts in both humans and domestic animals. It is therefore of great importance to determine ways to control A. flavus dissemination, survival and toxin formation. In this study we demonstrate the role of the veA regulatory gene in the contamination of peanut, corn and cotton by $A$. flavus. Virulence of $A$. flavus on peanut and corn seeds was reduced in the absence of the veA gene product. Generation of air-borne asexual spores was reduced and production of aflatoxin and sclerotia in peanut seeds, viable or non-viable, or in viable corn seed was completely blocked when infected with the $A$. flavus veA mutant ( $\Delta$ veA). In planta inoculation of cotton bolls also showed that conidiation was decreased in bolls inoculated with the $\Delta v e A$ strain and spread of the $\Delta v e A$ strain to seed in locules adjacent to the inoculated locule was less than observed with the wild-type veA strain. As observed in peanut and corn, no aflatoxin was produced in seed harvested from cotton bolls inoculated with the $\Delta v e A$ strain while aflatoxin was present in seed from wild-type veA inoculated bolls.
\end{abstract}

Keywords: Aflatoxin, Aspergillus flavus, veA, Seed infection.

\section{INTRODUCTION}

Aspergillus flavus is a soil-inhabiting, filamentous fungus that saprophytically utilizes a wide range of organic substrates. Though A. flavus is considered a saprophyte, it is also an opportunistic pathogen [1] that can invade agronomically important oil seed crops such as corn, peanut and cottonseed that are under biotic or abiotic stress. Among the toxic secondary metabolites produced by this organism are the aflatoxins (AFs) with aflatoxin B1 being the most potent natural carcinogen known [2-5]. AFs are polyketidederived compounds and the genes encoding enzymes involved in their biosynthesis are found clustered [6-8]. The adverse effects of AF contamination in crops such as corn can be much more serious in developing countries because in these regions the largest proportion of the crop is used for human consumption and there is a limited capacity to monitor the level of AF contamination in the grain [9]. Ingestion of food contaminated with high levels of AF has been implicated in acute toxicoses that often results in death while chronic, low-level exposure can lead to liver cancer, immune suppression, and stunting of growth in children among other pathological conditions $[10,11]$. The liver is the primary target organ in animals and humans where AFB1 is metabolized to the toxic and carcinogenic AFB1-epoxide form by cytochrome P450 enzymes [12-14]. From an economic perspective, the total annual loss to the U.S. corn industry alone has been estimated at approximately $\$ 200$ million. This includes losses from market rejections,

\footnotetext{
*Address correspondence to this author at the Department of Biological Sciences, Northern Illinois University, DeKalb, IL 60115, USA; Tel: (815) 753-0451; Fax: (815) 753-0461; E-mail: amcalvo@niu.edu
}

livestock losses, and the costs of AF sampling and testing [15]. Despite the many advances made in the biochemistry, genetics, and ecology of A. flavus and AF production, current control strategies fail to effectively eliminate AF contamination. Additional knowledge of the genetic mechanisms that control A. flavus morphogenesis, survival, and its ability to invade host tissues $[16,17]$ could lead to the development of novel biotechnological control strategies, contributing to the reduction of pre-harvest $\mathrm{AF}$ contamination.

A. flavus air-borne conidia (asexual spores) are an efficient means of dissemination for the fungus, leading to vast field infestations. In addition, under adverse conditions A. flavus is able to survive by forming numerous spherical, resistant structures called sclerotia [18-20]. High concentrations of AFs may occur in both conidia and sclerotia of $A$. flavus and certain toxicities associated with $A$. flavus infection have been attributed to the combined activities of AFs and other metabolites present in sclerotia [21]. Sclerotia are proposed to be vestigial forms of sexual cleisthothecia, produced by other Aspergillus spp (i.e. A. nidulans), that lost the capacity to produce spores [22]. For this reason, it is likely that genes homologous to those known to govern the formation of cleistothecia could be involved in the formation of sclerotia in A. flavus and other sclerotial-producing Aspergilli. Supporting this hypothesis, we have shown that the veA regulatory gene, required for cleistothecial production in A. nidulans, is also essential for sclerotial production in A. flavus and A. parasiticus [23-25]. Interestingly, veA has only been found in the fungal kingdom (Calvo, unpublished data). 
A number of studies have demonstrated that fungal morphogenesis is associated with natural product biosynthesis, including mycotoxin production (reviewed in $[26,27])$. We hypothesize the existence of common genetic regulatory mechanisms that govern both toxin production and morphological differentiation. In previous studies we have demonstrated that the same veA regulator that controls morphogenesis is also necessary for the production of several secondary metabolites, including $\mathrm{AF}$ in A. flavus and $A$. parasiticus [23-25], and sterigmatocystin (ST) in $A$. nidulans, where $\mathrm{ST}$ is the penultimate precursor in the $\mathrm{AF}$ biosynthetic pathway [28]. Interestingly, veA regulates the biosynthesis of other natural products indicating that veA is a global regulator of fungal morphological differentiation and secondary metabolism $[23,28]$.

Until now the role of $A$. flavus veA has been characterized on laboratory medium only. In this study we have taken the next step by evaluating the importance of $A$. flavus veA on the ability of the fungus to colonize and produce AF during growth on a variety of common, natural substrates such as peanut, corn and cottonseed.

\section{MATERIAL AND METHODS}

\section{Strains and Culture Conditions}

An Aspergillus flavus SRRC 70 isolate in which veA has been replaced by the $A$. parasiticus niaD marker gene ( $\triangle v e A:: n i a D$, referred to in this manuscript as $\Delta v e A$ ) [23] and an isogenic $A$. flavus 70 (pSL82) harboring a wild-type veA gene (referred to as veA+) were utilized in this study. The methods used to obtain the A. flavus $\Delta v e A$ strain used in this study are detailed in Duran et al., [23]. Three transformation controls $(v e A+)$ and five $\Delta v e A$ strains were obtained. Initial characterization of these strains showed that all $v e A+$ control strains had a consistent wild-type phenotype. The $\Delta v e A$ mutant phenotype was also consistent [23] and similar to those described in other Aspergillus spp. [24, 28]. Based on the consistency of these results, one representative $v e A+$ and $\Delta v e A$ strain were chosen for further studies, including functional genomics [29], and the present infection studies. Conidia of each culture were collected in $0.01 \%$ Tween 80 from point inoculated PDA plates that had been grown at $30^{\circ} \mathrm{C}$ in the light, a condition that promotes conidiation. Conidial suspensions were quantified with a hemacytometer under a light microscope and used as inoculum for the seed and plant infections.

\section{Seed Inoculations}

\section{Peanut}

Two peanut seed lines were used in this study, Flow Runner and Southernrunner (kindly provided by Dr. C. Holbrook, USDA, Georgia). The seeds were prepared following previously described procedures $[24,30]$. In brief, first, seeds were shelled, the cotyledons separated, and the embryo removed. Then seeds were weighed so that all the peanuts used were approximately $0.6 \mathrm{~g}$. For non-viable seed experiments, the cotyledons were autoclaved in a liquid cycle for $30 \mathrm{~min}$. For viable seed experiments, cotyledons were surfaced sterilized by immersion in $10 \%$ Clorox bleach for $1 \mathrm{~min}$ followed by two washes in sterile distilled water for $1 \mathrm{~min}$. Seeds were then inoculated with either the veA+ or $\Delta v e A$ strains and incubated as follows: For each strain, four peanut cotyledons were placed in sterile glass Petri dishes, resting on 3-MM chromatography paper (Whatman, Maidstone, England) saturated with sterile deionized water. The Petri dishes also contained a moisture reservoir with 5 $\mathrm{ml}$ of water. One hundred microliters containing $2.0 \times 10^{6}$ spores were spread on top of each cotyledon adaxial face. Cotyledons were incubated for 7 days at $30^{\circ} \mathrm{C}$ in the light $\left(25 \mu \mathrm{E} \mathrm{m}^{-2} \mathrm{~s}^{-1}\right)$. The experiment was repeated twice with similar results. To further demonstrate that the veA deletion is the sole cause of the phenotype observed in this study, additional peanut infection experiments were performed with two additional $v e A+$ control and $\Delta v e A$ mutants and consistent (no significant difference, $P<0.01$ ) results were obtained (data not shown). In all seed-inoculation experiments, Koch's postulates were determined after spore-counting to verify the identity of the Aspergillus strains infecting the seed.

\section{Corn}

Surface sterilized kernels of genotype B73 (a kind gift from Dr. P. Williams, USDA, Mississippi State) were immersed in a $0.01 \%$ Triton X-100 conidial suspension of either the $v e A+$ or $\Delta v e A$ strain $\left(5.0 \times 10^{6}\right.$ conidia per ml) and evaluated using a kernel screening assay (KSA) [31]. Briefly, following immersion in the fungal spore suspension, individual kernels were placed in plastic vial caps $(20 \mathrm{~mm}$ dia. $\mathrm{x} 6 \mathrm{~mm}$ height) that were positioned side by side in an open $60 \times 15 \mathrm{~mm}$ tissue culture plate bottom. One experimental unit consisted of four kernels in one plate bottom. A total of 10 culture plate bottoms containing four kernels each were placed in a clear tray $(243 \times 243 \times 18 \mathrm{~mm}$ Nunc bioassay tray, Thomas Scientific, Swedesboro, NJ) lined with 3-MM chromatography paper (Whatman). The lid was placed on top of the tray and high humidity was maintained by adding about $30 \mathrm{ml}$ of sterile deionized water to saturate the chromatography paper. Additional deionized water was added as needed during incubation to maintain humid conditions. The experiment was replicated 5 times. Kernels were incubated at $30^{\circ} \mathrm{C}$ in the light for 7 days after which they were removed from the trays for further analyses.

\section{In-Planta Cotton Assay}

Cotton plants (Gossypium hirsutum var. Coker 312) were greenhouse grown in 10-gal pots containing MetroMix 366 (Scotts). Plants were watered twice daily to field capacity and fertilized at planting with $15 \mathrm{~g}$ slow release Osmocote $(19 \% \mathrm{~N}: 6 \% \mathrm{P}: 12 \% \mathrm{~K}$; Scotts). Greenhouse temperatures ranged from $21-35^{\circ} \mathrm{C}$. Flowers were dated at opening and $24-25 \mathrm{dpa}$ bolls were inoculated with either the $v e A+$ or $\Delta v e A$ spore suspensions. Twenty cotton bolls present on greenhouse grown plants were wounded in the center of one of the locules to a depth of approximately $2 \mathrm{~mm}$ with a $3 \mathrm{~mm}$ diameter cork borer to simulate pink bollworm exit holes. Approximately $10^{4}$ conidia in $10 \mu 1$ of $0.01 \%$ Triton X-100 were inoculated at the wound site and the fungus allowed to colonize the bolls for three weeks. Ten bolls inoculated with either of the fungi were collected and analyzed for fungal spread and aggressiveness. Fungal spread from the inoculated locule to the adjacent locules was measured by the amount of ergosterol present in the seed of the adjacent locules. Intercarpellary membranes adjacent to the inoculated locule were examined to measure fungal aggressiveness [32]. Membranes were rated as follows; 0, no symptoms; 1, 
discolored on the surface next to the inoculated locule; 2 , same as a rating of 1 plus slightly discolored on the opposite face; 3, highly discolored on both faces; 4 , same as 3 plus pinhole tears; 5 , same as 3 plus $1 \mathrm{~mm}$ or larger tears.

\section{Conidiation and Sclerotial Formation Studies}

Conidia were released from the infected seeds by adding $3 \mathrm{ml}$ of Tween 80 water and vortexing for one min. Conidia were counted with a hemacytometer (Hausser Scientific, Horsham, PA). Four peanut cotyledons and 8 corn seeds were used for counting per treatment. Sclerotia were visualized with a Leica MZ75 dissecting microscope. Micrographs were obtained using Leica MZ75 microscope attached to a digital camera (Spot Insight color Model \#3.2.0, Diagnostic Instruments, Sterling Heights, Michigan).

\section{AF Analysis}

Seeds (4 infected peanut cotyledons, 8 corn kernels, or 8 cottonseeds) were ground in liquid nitrogen. Ground material was added to $50 \mathrm{~mL}$ of sterile water. Then $25 \mathrm{~mL}$ of acetone was added to each sample and shaken on a rotary platform at room temperature for $1 \mathrm{~h}$. Each sample was filtered through Whatman paper and added to a $250 \mathrm{~mL}$ separatory funnel. Seventy $\mathrm{mL}$ of methylene chloride $(\mathrm{MeCl})$ was added to the funnel and inverted three times. The liquid phases were allowed to separate. A fluted funnel containing a filter filled with sodium sulfate was place in a beaker to absorb the remaining water. The bottom layer in the separatory funnel was released through the sodium sulfate and collected in the beaker. Liquid extracts were allowed to dry overnight. Dried extracts were then resuspended in $2 \mathrm{~mL}$ of $\mathrm{MeCl}$, and transferred to a $2 \mathrm{~mL}$ tube, allowed to dry once again and the residue was resuspended in acetone and spotted on a TLC plate (Si250F, J.T. Baker). The plate was then developed in toluene/ethyl acetate/formic acid $(5: 4: 1, \mathrm{v} / \mathrm{v} / \mathrm{v})$, and air-dried. The TLC plates were sprayed with aluminum chloride (15\% in ethanol) to intensify AF fluorescence upon exposure to long-wave (365-nm) UV light and baked for $10 \mathrm{~min}$ at $80^{\circ} \mathrm{C}$ prior to being viewed. The approximate sensitivity of the assay was $25 \mathrm{ng}$. AF purchased from Sigma-Aldrich (St. Louis) was used as a standard.

\section{Ergosterol Analysis}

The ergosterol content of peanut seeds (cotyledons) was analyzed as a quantitative measure of fungal growth in the colonized plant material (in addition to the micrographs shown). Ground seeds (1g) were extracted overnight at room temperature in $4 \mathrm{~mL}$ of 2:1 chloroform:methanol, filtered through sterile miracloth, dried and resuspended in $3 \mathrm{~mL}$ of the same extraction mixture. Ergosterol analysis was performed by injecting $25 \mu \mathrm{l}$ of the suspension into a Waters 1525 HPLC system equipped with a binary pump and a Waters 717 Autosampler. HPLC separation was performed at

$50^{\circ} \mathrm{C}$ on a Phenomenex $\mathrm{C} 184.6 \times 25 \mathrm{~mm}, 5$ micron analytical column with a column guard. UV detection was at $282 \mathrm{~nm}$ (Waters 2487 Dual $\lambda$ Absorbance Detector). Compounds were eluted using an isocratic mobile phase of $100 \%$ HPLCgrade methanol with a flow rate of $1.0 \mathrm{ml} / \mathrm{min}$. Ergosterol quantification of the samples was determined by comparing peak areas of samples to a standard curve created using HPLC-grade ergosterol (Sigma-Aldrich, St. Louis).

\section{Statistical Analysis}

Quantititative data was evaluated by analysis of variance (ANOVA), in conjuction with the Tukey's test for multiple comparisons using the Statistical Package for the Social Sciences (SPSS 15.0 for windows SPSS Inc. Chicago, IL). Fungal discoloration and damage to intercarpellary membranes flanking the infected locules of 10 bolls were assessed and analyzed by Student t-test using GraphPad Prism software. Differences among mean comparisons between the $\triangle v e A$ and $v e A+$ values were significant $(\mathrm{P}<0.05)$ according to the Wilcoxin signed-rank test.

\section{RESULTS AND DISCUSSION}

It is well known that asexual spores are an efficient means of fungal dissemination [33, 34], causing infestation of field crops, as is the case for AF-producing isolates of $A$. flavus. In addition, this fungus produces resistant structures termed sclerotia that are able to survive adverse environmental conditions over several years [18-20] and can contain high levels of AF [35]. When environmental conditions are again favorable, sclerotia will produce mycelia and conidia thus reestablishing infections and AF contamination of food commodities. In this study we investigated the importance of the global regulatory gene $v e A$ in developmental differentiation, AF production and virulence of $A$. flavus when growing on a variety of oil seeds that are common hosts for this organism. The veA gene product is unique to fungi and conserved in Aspergilli and across fungal genera (Calvo et al., unpublished). We are evaluating the suitability of $v e A$ as a possible target for future control strategies to reduce fungal infection and toxin contamination of important agricultural commodities.

\section{Peanut Infection}

All strains were able to grow on peanut seeds, however, we found that peanut seed infection by the $v e A+$ strain was significantly different when compared to seed infected with the $\Delta v e A$ strain. Conidial formation was reduced in the case of peanut seeds inoculated with $A$. flavus $\Delta v e A$ in comparison with seeds infected with the veA+ strain. This was true when the strains were growing on Flowrunner or Southernrunner peanut lines, with conidiation reduced to $42 \%$ and $3 \%$ on viable seeds and to $67 \%$ and $57 \%$ on nonviable seeds, respectively (Figs. 1, 2A, D). Fungal colonization of the seeds was reduced in the absence of the $v e A$ gene in either viable or non-viable seeds as determined by micrographs as well as ergosterol levels measured in the infected seeds (Figs. 1, 2B, E). The presence of fungi in commodities such as corn and peanut can be determined by measuring ergosterol which is the predominant sterol in most fungi and not found in plants [36]. Fungal ergosterol content in viable Flowrunner and Southernrunner peanut seeds infected with the $\Delta v e A$ mutant were reduced to $19 \%$ and $29 \%$ (respectively) as compared to levels obtained in seeds infected with the $v e A+$ strain. In $\Delta v e A$-infected non-viable seed the percentage of ergosterol detected was reduced to $45 \%$ and $73 \%$ in Flowrunner and Southernrunner respectively, compared to the levels observed in veAtinfected seeds (Fig. 2B, E). Our results indicate that the presence of a wild-type allele of $A$. flavus veA is necessary to establish normal virulence levels in peanut, since its absence results in reduced growth and impaired developmental processes in this agronomically important plant pathogen. 


\section{A}

\section{Flowrunner}

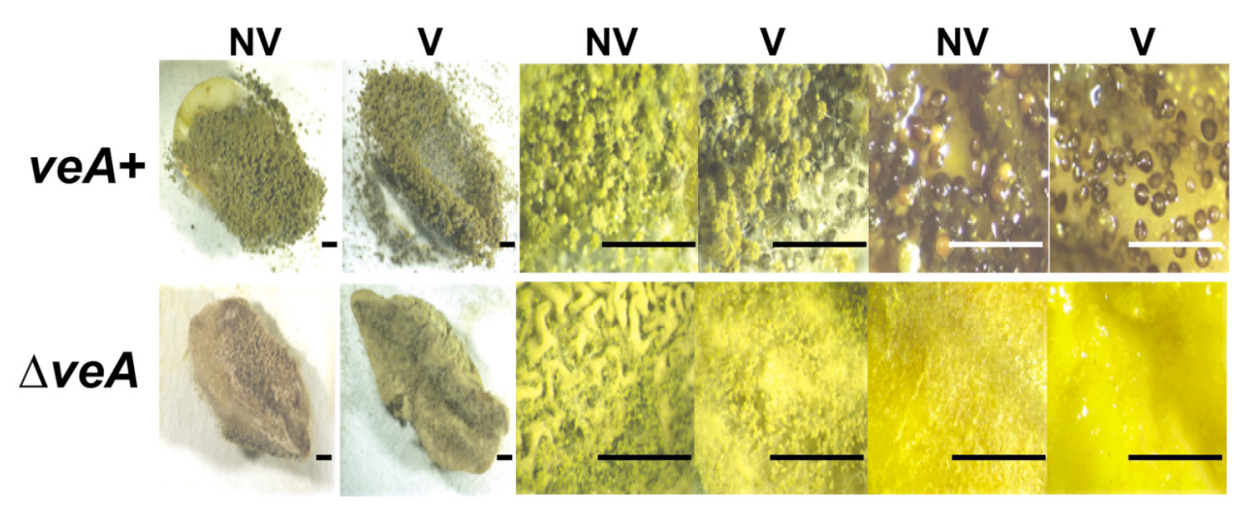

B

\section{Southernrunner}

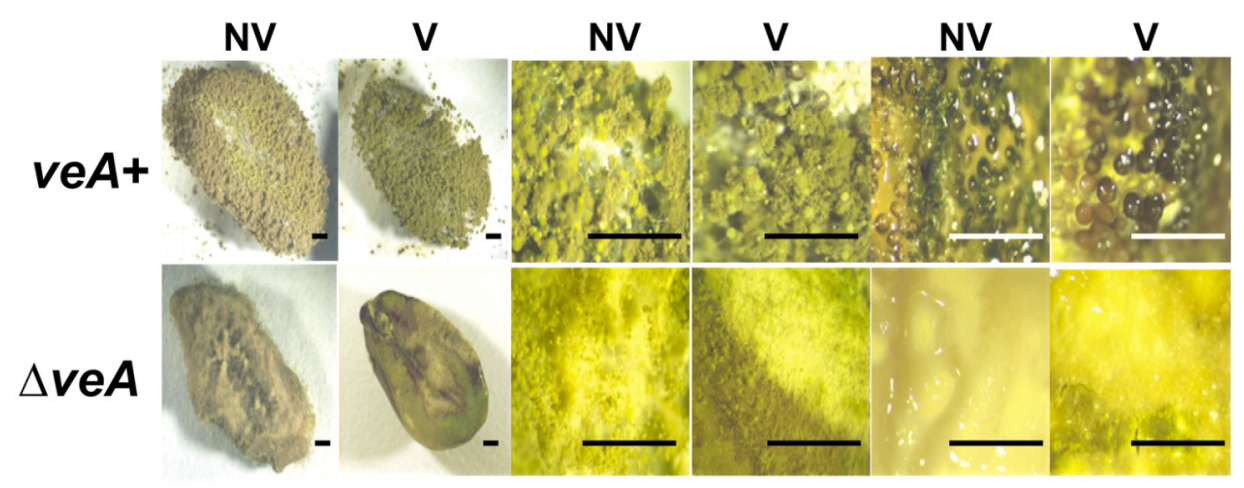

Fig. (1) Peanut seed lines contaminated by A. flavus wild-type veA+ strain (WT) or deletion veA ( $\Delta$ veA) strain. (A) Flowrunner seed line; (B) Southernrunner seed line. Non-viable (NV) or viable (V) peanut cotyledons. Micrographs were captured with a Leica MZ75 dissecting microscope. The last four panels on the right side (in both $\mathbf{A}$ and $\mathbf{B}$ ) correspond to samples that were sprayed with $70 \%$ ethanol to flatten conidiophores for better visualization of sclerotia. Bars represent $2 \mathrm{~mm}$.

The $\Delta v e A$ mutant is compromised in its capacity to colonize seeds as determined by micrographs, ergosterol levels and reduced conidiation (Figs. 1, 2). This is particularly notable in viable seed assays, exemplified in this peanut seed experiment. In general, viable seeds were more resistant to A. flavus infection than non-viable seeds in both peanut lines (Fig. 2). Plants have stress-response defense mechanisms that can be activated by different factors, including fungal attack (i.e. [37, 38]). As in our A. flavus study, differences between viable and non-viable peanut seeds infected with $A$. parasiticus have been previously interpreted to be a consequence of differences in the effectiveness of the plant defensive response when infected by the fungus [30]. Our results also indicate that $v e A+$ in $A$. flavus is especially relevant for colonization of viable seeds, as shown in both peanut lines tested (Figs. 1, 2).

Sclerotial production was completely blocked in the $\Delta v e A$-inoculated Flowrunner or Southernrunner peanut seeds, while numerous sclerotia, tightly adhered to the seed surface, were found in seeds inoculated with the veA+ strain. This demonstrates that the veA gene is necessary for sclerotial formation in $A$. flavus when growing on peanut seeds (Fig. 1).
Importantly, our AF analysis of peanut seeds indicated that this mycotoxin was absent from all seeds, viable or nonviable, when infected with the A. flavus $\Delta v e A$ mutant, while AF was detected when seeds were infected with the veA+ strain (Figs. 2C, F). This indicates that a functional veA gene is necessary for AF production during growth on a natural substrate such as peanut seed.

\section{Corn Infection}

Visual examination corn kernels incubated with illumination revealed a higher level of colonization and conidiation present on the surface of kernels inoculated with the veA+ strain than with the $\triangle v e A$ strain. This was confirmed by counts of conidia (Fig. 3B). Kernels inoculated with the $\triangle v e A$ strain showed a reduction in conidial production of about $11 \%$ with respect to the conidia produced by the $v e A+$ strain. Interestingly, the number of conidia increased in the $\triangle v e A$ strain compared to the veA+ strain when grown on artificial medium ([23] and Fig. 3B). In previous studies in the model fungus $A$. nidulans we showed that conidiation levels in the $\triangle v e A$ strain were medium-dependent [28]. The results of our current study 
A

Flowrunner

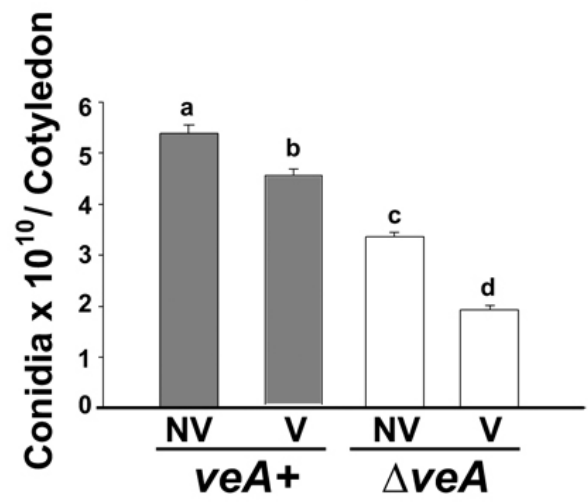

B

Flowrunner

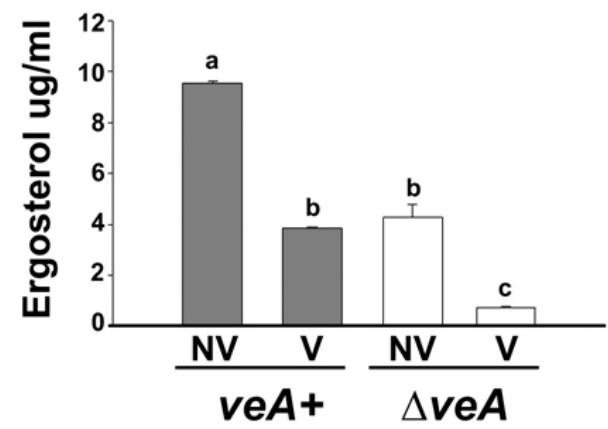

C

Flowrunner

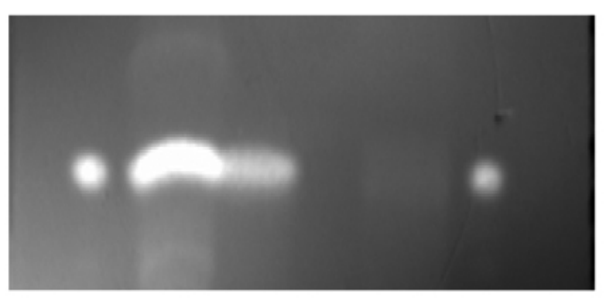

Std. NV V NV V Std.
D

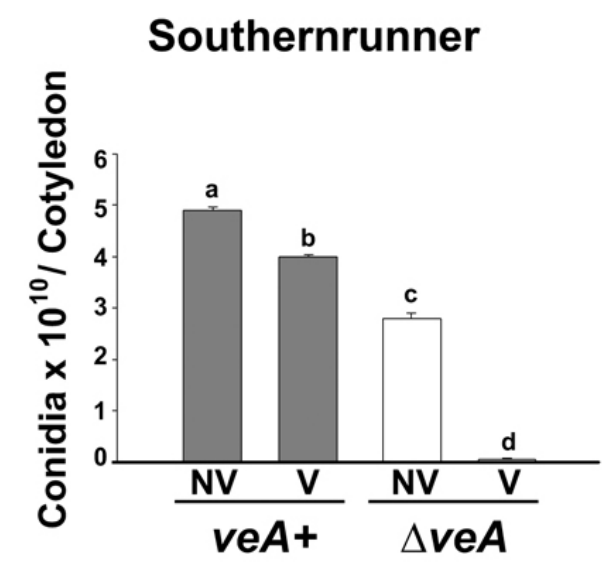

E

Southernrunner

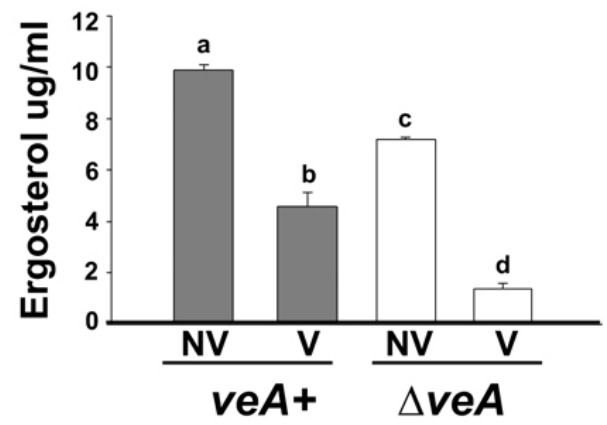

$\mathbf{F}$

\section{Southernrunner}

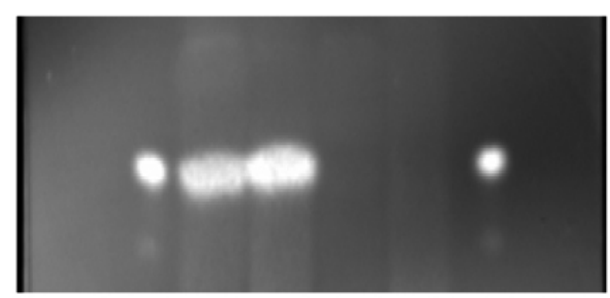

Std. NV V NV V Std.

$v e A+\Delta v e A$

Fig. (2). Comparison of the effects of A. flavus veA deletion on the colonization between two peanut lines Flowrunner and Southernrunner. (A, D) Quantification of conidial production by $A$. flavus wild-type veA+ strain (WT) or deletion veA $(\Delta v e A)$ strain when infecting Flowrunner peanut seeds (A) or Southernrunner seed line (D). (B, E) Quantification of fungal ergosterol content in infected peanut cotyledons of Flowrunner peanut line (B) and Southernrunner line (E). (C, F) TLC analysis of AF content in infected Flowrunner cotyledons (C) or Southernrunner cotyledons (F). non-viable (NV) or viable (V). Std.: AF standard. The values represent means of 4 replicates. Standard error is shown. Columns with different letters represent values that are statistically significantly different $(P<0.05)$. 


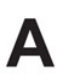

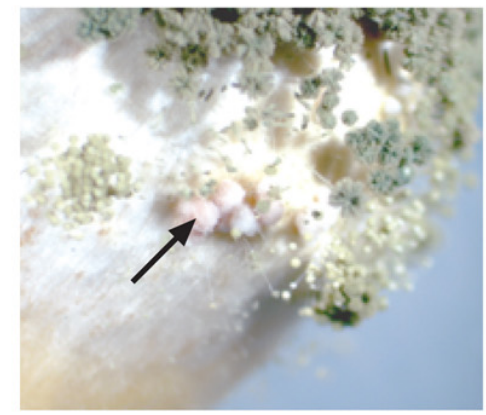

veA+

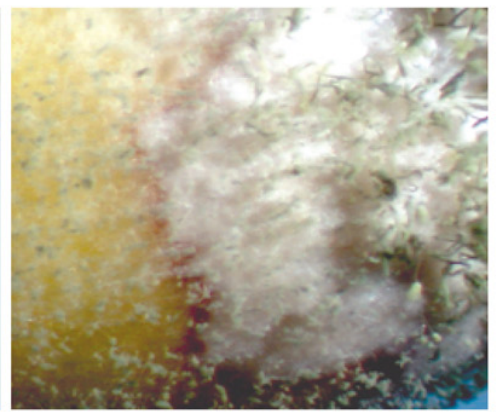

$\Delta v e A$
B

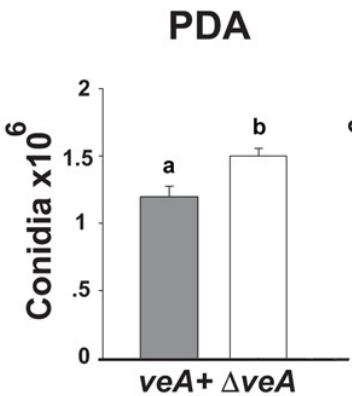

Corn Kernels

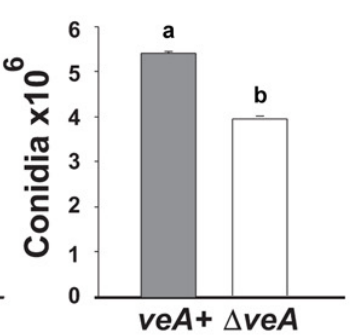

Corn Kernels

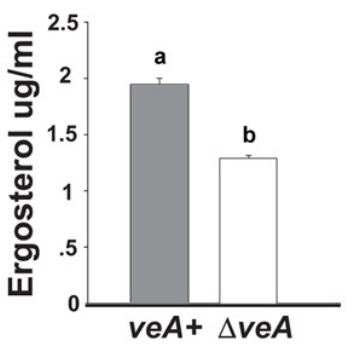

\section{C}

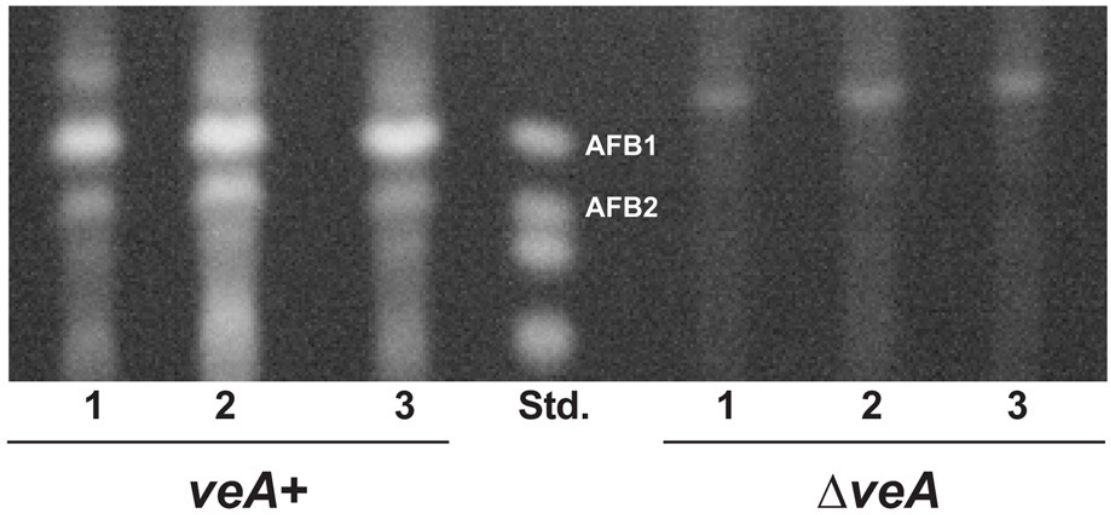

Fig. (3). Effect of the veA deletion on A. flavus colonization of corn kernels. (A) Presence of non-melanized sclerotia on surface of veA+ inoculated corn kernel (arrow denotes cluster of sclerotia) and absence of sclerotia on the surface of a $\Delta v e A$ inoculated kernel. (B) Conidial production of $A$. flavus $v e A+$ and $\Delta v e A$ strains on corn kernels (per 8 kernels) and on PDA medium (per 5 mm-diameter core). In (B) columns with different letters represent values that are statistically significantly different $(\mathrm{P}<0.05)$. (C) Thin Layer Chromatography analysis of AF production by $A$. flavus veA+ and $\Delta v e A$ strains growing on corn kernels. The experiment included five replicates.

suggest that this is also the case in the Alavus $\Delta v e A$ strain, in which conidiation is severely affected when growing on natural substrates such as peanut and corn seeds. This is further supported by a previous study of $A$. parasiticus growth on peanut seeds from our laboratory [24]. In the model fungus $A$. nidulans it is known that the role of $v e A$ in regulating conidiation is influenced by external stimuli (i.e. light) $[25,39]$. The $A$. nidulans veA gene product (VeA) forms a nuclear protein complex with several proteins, including light-sensing proteins and proteins involved in regulating development [40]. We hypothesize that VeA could act as a scaffold protein that integrates external stimuli with a genetic response by an orchestrated action with VeAinteracting proteins, leading to developmental adaptative responses [25]. Based on this and previous studies [24, 28], it is possible that VeA function in Aspergilli could be influenced by other environmental cues, in addition to light, for example, availability of different types of nutrients and/or in this case unknown factors generated during seed colonization that could lead to the observed increase in conidiation. 


\section{A}
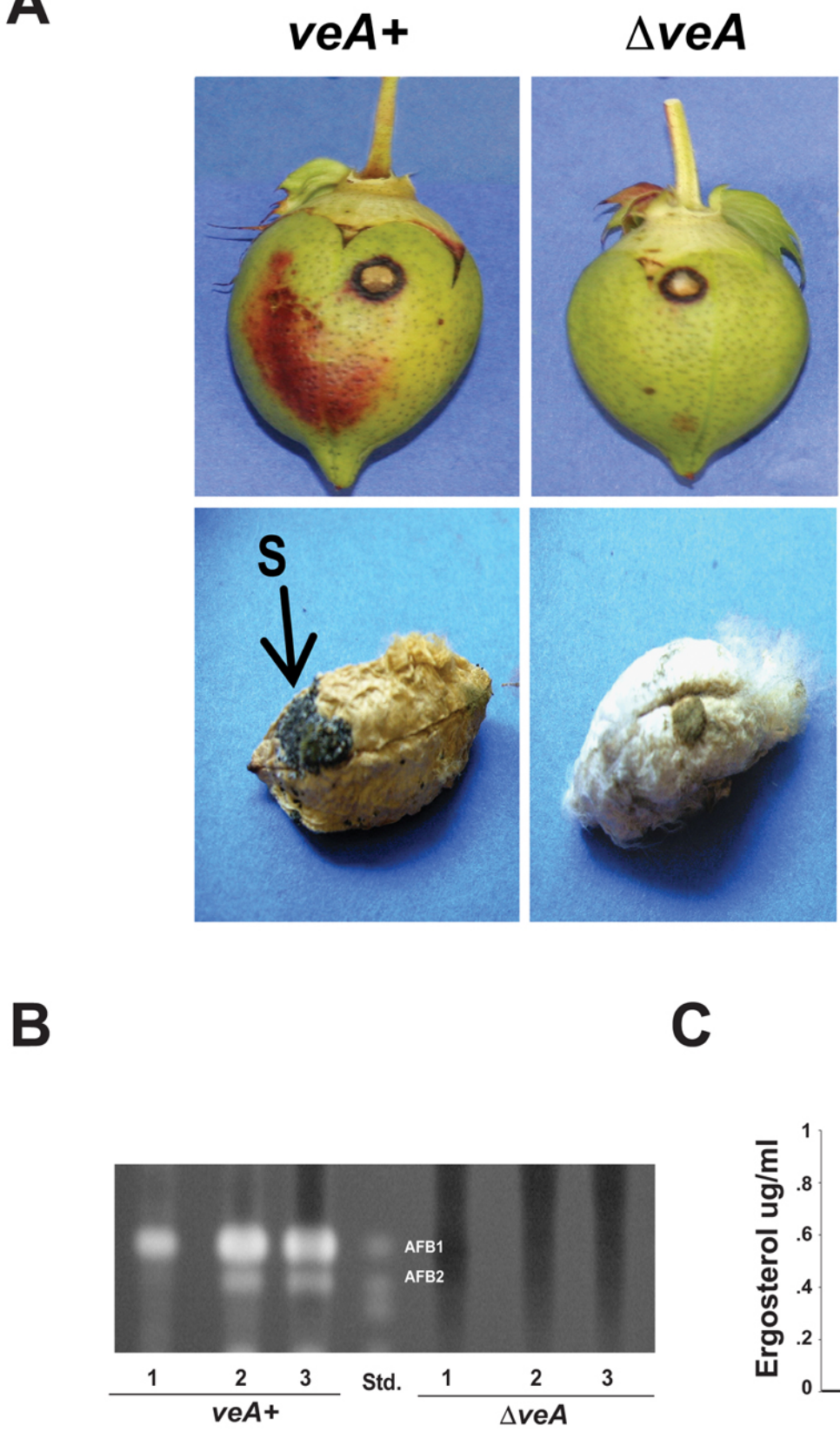

\section{Cotton}

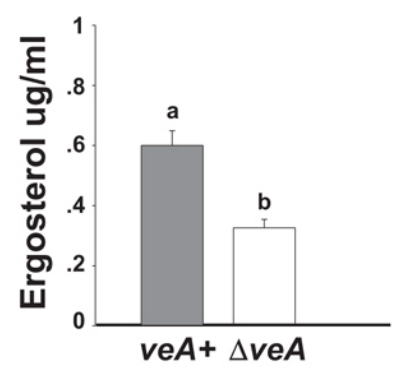

Fig. (4). Effect of veA deletion on A. flavus colonization of cotton bolls. (A) Top Panels: Outer surface of boll near inoculation site demonstrating significant discoloration following inoculation with the $v e A+$ strain and little to no discoloration on the $\Delta v e A$ inoculated boll. Lower Panels: Surface of locule from boll inoculated with the $v e A+$ strain demonstrating presence of melanized sclerotia (S; denoted by arrow) and a locule from a boll inoculated with the $\Delta v e A$ strain lacking sclerotia. (B) TLC analysis of AF production from extracts of cottonseed harvested from bolls inoculated with either the veA+ or $\Delta v e A$ strain. Experiments were repeated twice with similar results. (C) Quantification of ergosterol content from seed harvested from locules adjacent to the inoculated locule. In (C) columns with different letters represent values that are statistically significantly different $(P<0.05)$.

As in the case of the peanut infection studies, ergosterol level were also significantly reduced when comparing kernels infected with $\Delta v e A$ and the $v e A+$ control (Fig. 3B), indicating a reduction in fungal colonization of seeds in the absence of $v e A$.

As was observed with peanut seed (above), sclerotia were present on surfaces of corn kernels inoculated with the veA+ strain while no sclerotia were detected on seeds inoculated with the $\Delta v e A$ strain (Fig. 3A). Our analyses also demonstrated that $\mathrm{AF}$ was produced in corn kernels inoculated with the veA+ strain, while no $\mathrm{AF}$ was detected with the $\Delta v e A$ strain (Fig. 3C).

\section{In Planta Cotton Infection}

Visual examination of the cotton bolls showed that there was more discoloration on the boll surface for those bolls inoculated with the $v e A+$ strain compared to the $\Delta v e A$ strain (Fig. 4A). The significance of this discoloration is not clear 
at this time but may be related to stress. There were more conidia present on locules inoculated with the veA+ strain compared to the $\Delta v e A$ strain (Fig. 4A). As expected, there were no sclerotia present on the locules of bolls inoculated with the $\Delta v e A$ strain while sclerotia were evident in locules from bolls inoculated with the veA+ strain (Fig. 4A). As in previous observations with peanut and corn seeds, A. flavus $\Delta v e A$-infected cotton seeds were free of AF contamination, while $\mathrm{AF}$ accumulated in those seeds infected with the veA+ strain (Fig. 4B). A method for evaluating fungal spread between cotton boll locules is to look for the presence of bright greenish yellow fluorescence (BGYF) on cotton lint as this has been shown to be a reliable indicator of $A$. flavus spread [41]. However, this was not possible in this study as BGYF is a metabolite of the oxidation of kojic acid and the $\Delta v e A$ strain does not appear to produce kojic acid during growth in cotton bolls. Copius amounts of BGYF was detected on the lint of locules adjacent to the locule inoculated with the $v e A+$ strain (data not shown). The degree of fungal spread was instead determined by analysis of ergosterol levels in the seed collected from locules adjacent to the inoculated locule. Ergosterol levels were significantly greater in the locules taken from bolls infected with the veA+ strain compared to those infected with the $\Delta v e A$ strain (Fig. 4C). The degree of aggressiveness of the fungal strains was determined by microscopic examination of discoloration and damage to the intercarpellary membranes of the locules adjacent to the inoculated locule (Fig. 5). The incidence of greater discoloration and tears on the side of the intercarpellary membrane opposite the inoculated locule was consistently greater in the $v e A+$ inoculated bolls compared to those inoculated with the $\Delta v e A$ strain. This was supported by statistical analysis that indicated the $v e A+$ strain produced a greater degree of membrane discoloration and tearing $(P<$ 0.05 ) than bolls inoculated with the $\Delta v e A$ strain (Fig. 5). The increased level of spread and aggressiveness of the veA+ strain would indicate that it is more efficient at penetrating the intercarpellary wall separating the locules. This requires the production of a number of different hydrolytic enzymes such as pectinases and proteases, suggesting that their production may be either directly or indirectly dependent on VeA. Future studies in our laboratory will be directed at elucidating the possible role of veA in the production of hydrolytic enzymes implicated in plant tissue colonization.
INNER

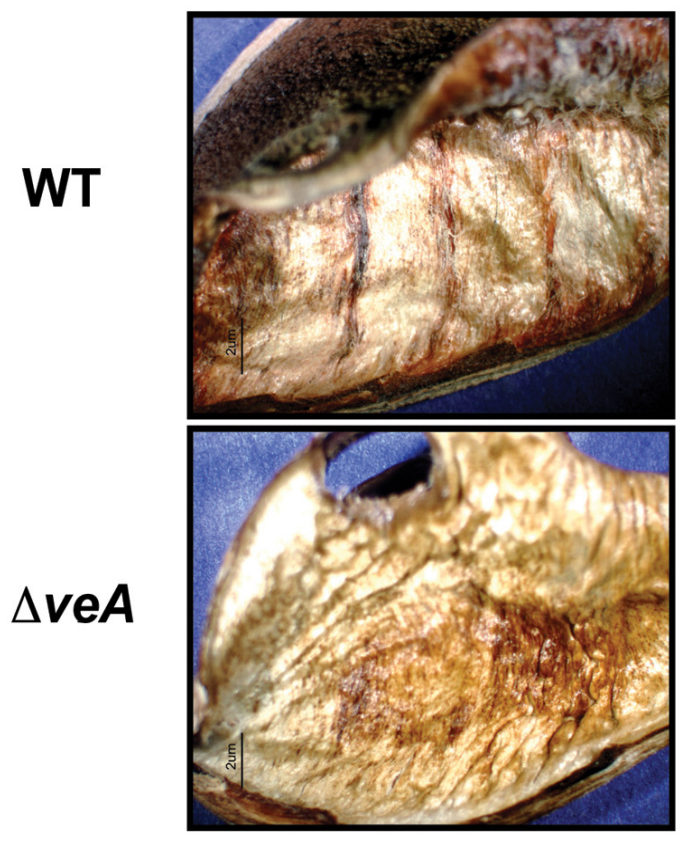

OPPOSITE

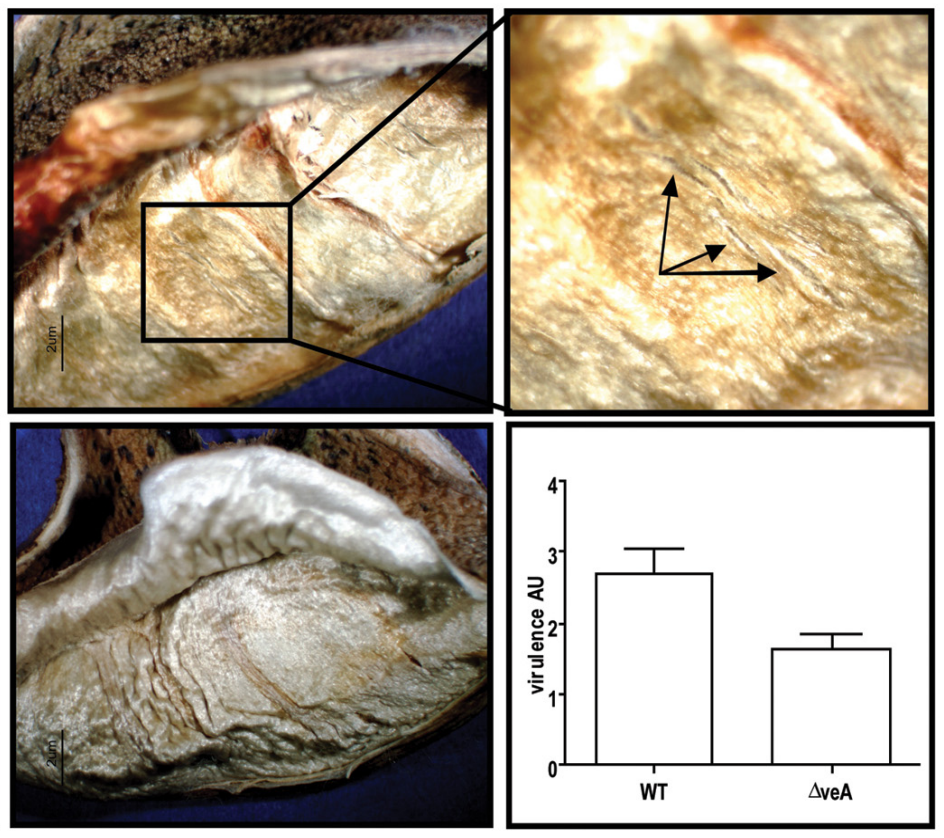

Fig. (5). Analysis of intercarpellary membrane damage from cotton bolls infected with either $A$. flavus veA+ or $\Delta v e A$ mutant strains. Upper left panel: Micrograph demonstrating discoloration on the side of the membrane facing the inoculated locule of a boll infected with the $v e A+$ strain. Upper middle panel: Micrograph demonstrating discoloration on the opposite side of the membrane adjacent to the inoculated locule of a boll infected with the $v e A+$ strain. Upper right panel: enlargement of area of membrane denoted in middle panel. Note tears in the membrane indicative of extensive damage by the fungus. Lower left panel: Micrograph demonstrating discoloration on the side of the membrane facing the inoculated locule of a boll infected with the $\Delta v e A$ strain. Lower middle panel: Micrograph demonstrating lack of discoloration and damage on the opposite side of the membrane adjacent to the inoculated locule of a boll infected with the $\Delta v e A$ strain. Lower right panel: Values represent a Student t-test analysis of the means and standard deviations of damage observations taken from the 2 intercarpellary membranes flanking the infected locules of 10 bolls. Differences among mean comparisons between the $\Delta v e A$ and $v e A+$ samples were statistically significant $(P<0.05)$ according to the Wilcoxin rank test. Membranes were rated as follows: 0 , no discoloration; 1 , discolored on surface of membrane facing inoculated locule; 2, same as 1 plus slightly discolored on opposite surface; 3, highly discolored on both surfaces; 4, same as 3 but presence of pin-hole tears; 5, same as 3 plus tears of $1 \mathrm{~mm}$ or larger. Experiments were repeated twice with similar results. 


\section{CONCLUSIONS}

This study has shown how a functional veA contributes to the virulence of $A$. flavus during in vitro infection of peanut and corn seed and in planta infection of cottonseed and boll tissues. A. flavus conidiation, sclerotial production, and AF biosynthesis are severely compromised when the fungus lacks a functional veA gene product. In this study it is particularly interesting that seed substrates, as opposed to laboratory media, promoted A. flavus conidiation in the presence of a veA wild-type allele. Furthermore, our study demonstrated that this holds true during the infection of a number of different oil seeds both in vitro and in planta. The observation that veA can play a role as a virulence factor during $A$. flavus invasion of crops, together with the fact that $v e A$ has only been found in fungi, suggest a high potential for the implementation of a control strategy targeting $v e A$ to inhibit $A$ flavus invasion of food and feed crops. Since veA is conserved in other fungi, such a strategy could also potentially be used to control contamination of crops by other fungal pathogens.

\section{ACKNOWLEDGMENTS}

We thank Dr. Corley Holbrook USDA-ARS, CGBRU and Dr. P. Williams, USDA, Mississippi State) for providing the peanut and corn seed (respectively). This work was funded by Northern Illinois University. JWC acknowledges Ms. Pamela Harris-Coward and Mr. Hieu Nguyen for their assistance with analyses of corn and cotton boll infection and $\mathrm{AF}$ production.

\section{REFERENCES}

[1] Mellon JE, Cotty PJ, Dowd MK. Aspergillus flavus hydrolases: their roles in pathogenesis and substrate utilization. Appl Microbiol Biotechnol 2007; 77: 497-504.

[2] Payne GP, Brown MP. Genetics and physiology of aflatoxin biosynthesis. Annu Rev Phytopathol 1998; 36: 329-62.

[3] Sweeney MJ, Dobson AD. Molecular biology of Mycotoxin biosynthesis. FEMS Microbiol Lett 1999; 175: 149-63.

[4] Trail F, Mahanti N, Linz J. Molecular biology of aflatoxin biosynthesis. Microbiology 1995; 141: 755-65.

[5] Squire RA. Ranking animal carcinogens: a proposed regulatory approach. Science 1981; 214: 877-80.

[6] Yabe K, Nakajima H. Enzyme reactions and genes in aflatoxin biosynthesis. Appl Microbiol Biotechnol 2004; 64: 745-55.

[7] Yu J, Chang PK, Ehrlich KC, et al. Clustered pathway genes in aflatoxin biosynthesis. Appl Environ Microbiol 2004; 70: 1253-62.

[8] Bennett JW, Klich M. Mycotoxins. Clin Microbiol Rev 2003; 16 : 497-516.

[9] Widstrom NW. The aflatoxin problem with corn grain. Adv Agron 1996; 56: 219-80.

[10] Turner PC, Moore SE, Hall AJ, Prentice AM, Wild CP. Modification of immune function through exposure to dietary aflatoxin in Gambian children. Environ Health Perspect 2003; 111: 217-20.

[11] Hsieh DP. Potential human health hazards of mycotoxins. In: Natori SHK, Ueno Y, Eds. Mycotoxins and phytotoxins: Amsterdam: Elsevier 1988; pp. 69-80.

[12] Cary JW, Linz JE, Bhatnagar D. Microbial foodborne diseases: mechanisms of pathogenesis and toxin synthesis, In: Cary JW, Linz JE, Bhatnagar D, Eds. Aflatoxins: biological significance and regulation of biosynthesis. Technomic Publishing Co.: Lancaster, PA 2000; pp. 317-61.

[13] Dvorackova I, Kusak V. Hepatocellular carcinoma (a 28-year necropsy review). J Environ Pathol Toxicol Oncol 1990; 10: 220-4.
[14] Wogan GN. Aflatoxins as risk factors for hepatocellular carcinoma in humans. Cancer Res 1992; 52: 2114s-2118s.

[15] Wu F. The true economic impact of aflatoxin in U.S. crops. In: Proceeding of the 2006 Annual Multi-Crop Aflatoxin/Fumonisin Elimination \& Fungal Genomics Workshop. Fart Warth, Texas Oct 16-18 2006; p.49.

[16] Bhatnagar D, Cary JW, Ehrlich K, Yu J, Cleveland TE. Understanding the genetics of regulation of aflatoxin production and Aspergillus flavus development. Mycopathologia 2006; 162: 155-66.

[17] Brown RL, Bhatnagar D, Cleveland TE, Cary JW. Recent advances in preharvest prevention of mycotoxin contamination. In: Sinha KK, Bhatnagar D, Eds. Mycotoxin in agriculture and food safety. New York: Marcel Dekkar, Inc 1998; pp. 351-79.

[18] Coley-Smith JR, Cooke RC. Survival and germination of fungal sclerotia. Annu Rev Phytopathol 1971; 9: 65-92.

[19] Malloch D, Cain RF. The Trichocomataceae: ascomycetes with Aspergillus, Paecilomyces and Penicillium imperfect states. Can J Bot 1972; 50: 2613-28.

[20] Wicklow DT. Survival of Aspergillus flavus sclerotia in soil. Trans Br Mycol Soc 1987; 89: 131-4.

[21] Wicklow DT, Cole RJ. Tremorgenic indole metabolites and aflatoxins in sclerotia of Aspergillus flavus: an evolutionary perspective. Can J Bot 1982; 60: 525-8.

[22] Geiser DM, Timberlake WE, Arnold ML. Loss of meiosis in Aspergillus. Mol Biol Evol 1996; 13: 809-17.

[23] Duran R, Cary J, Calvo A. Production of cyclopiazonic acid, aflatrem, and aflatoxin by Aspergillus flavus is regulated by veA, a gene necessary for sclerotial formation. Appl Microbiol Biotechnol 2007; 73: 1158-68.

[24] Calvo AM, Bok J-W, Brooks W, Keller NP. VeA is required for toxin and sclerotial production in Aspergillus parasiticus. Appl Environ Microbiol 2004; 70: 4733-9.

[25] Calvo AM. The VeA regulatory system and its role in morphological and chemical development in fungi. Fungal Genet Biol 2008; 45: 1053-61.

[26] Shwab EK, Keller NP. Regulation of secondary metabolite production in filamentous ascomycetes. Mycol Res 2008; 112: 22530.

[27] Calvo AM, Wilson RA, Bok JW, Keller NP. Relationship between secondary metabolism and fungal development. Microbiol Mol Biol Rev 2002; 66: 447-59.

[28] Kato N, Brooks W, Calvo AM. The expression of terigmatocystin and penicillin genes in Aspergillus nidulans is controlled by veA, a gene required for sexual development. Eukaryot Cell 2003; 2: 1178-86.

[29] Cary J, Obrian G, Nielsen D, et al. Elucidation of veA -dependent genes associated with aflatoxin and sclerotial production in Aspergillus flavus by functional genomics. Appl Microbiol Biotechnol 2007; 76: 1107-18.

[30] Wilson RA, Calvo AM, Chang PK, Keller NP. Characterization of the Aspergillus parasiticus delta12-desaturase gene: a role for lipid metabolism in the Aspergillus-seed interaction. Microbiology 2004; 150: 2881-8.

[31] Brown RL, Cleveland TE, Payne GA, Woloshuk CP, Campbell $\mathrm{KW}$, White DG. Determination of resistance to aflatoxin production in maize kernels and detection of fungal colonization using an Aspergillus flavus transformant expressing Escherichia coli b-glucuronidase. Phytopathology 1995; 85: 983-9.

[32] Sheih M-T, Brown RL, Whitehead MP, et al. Molecular genetic evidence for the involvement of a specific polygalacturonase, P2c, in the invasion and spread of Aspergillus flavus in cotton bolls. Appl Environ Microbiol 1997; 63: 3548-52.

[33] Adams TH, Wieser JK, Yu JH. A sexual sporulation in Aspergillus nidulans. Microbiol Mol Biol Rev 1998; 62: 35-54.

[34] Horn BW. Biodiversity of Aspergillus section Flavi in the United States: a review. Food Addit Contam 2007; 24: 1088-101.

[35] Wicklow DT, Shotwell OL. Intrafungal distribution of aflatoxins among conidia and sclerotia of Aspergillus flavus and Aspergillus parasiticus. Can J Microbiol 1983; 29: 1-5.

[36] Seitz LM, Mohr HE, Burroughs R, Sauer B. Ergosterol as an indicator of fungal invasion in grains. Cereal Chem 1977; 54: 1207-17. 
[37] Gao X, Shim WB, Gobel C, et al. Disruption of a maize 9-lipoxygenase results in increased resistance to fungal pathogens and reduced levels of contamination with mycotoxin fumonisin. Mol Plant Microbe Interact 2007; 20: 922-33.

[38] Gao X, Stumpe M, Feussner I, Kolomiets M. A novel plastidial lipoxygenase of maize (Zea mays) ZmLOX6 encodes for a fatty acid hydroperoxide lyase and is uniquely regulated by phytohormones and pathogen infection. Planta 2008; 227: 491-503.
[39] Purschwitz J, Muller S, Kastner C, et al. Functional and physical interaction of blue- and red-light sensors in Aspergillus nidulans. Curr Biol 2008; 18: 255-9.

[40] Bayram O, Krappmann S, Ni M, et al. VelB/VeA/LaeA complex coordinates light signal with fungal development and secondary metabolism. Science 2008; 320: 1504-6.

[41] Cotty PJ. Virulence and cultural characteristics of two Aspergillus flavus strains pathogenic on cotton. Phytopathology 1989; 79: 80814

(C) Duran et al.; Licensee Bentham Open.

This is an open access article licensed under the terms of the Creative Commons Attribution Non-Commercial License (http://creativecommons.org/licenses/by$\mathrm{nc} / 3.0 /$ ) which permits unrestricted, non-commercial use, distribution and reproduction in any medium, provided the work is properly cited. 